\title{
Biosynthèse de l'ergostérol par Penicillium caseicolum
}

par

\author{
A. HUYGHEBAERT* et H. DE MOOR*
}

\section{INTRODUCTION}

Les procédés technologiques, appliqués dans l'industrie laitière, ont une influence plus ou moins prononcée sur les composants de la matière grasse du lait. Les modifications qualitatives et quantitatives de plusieurs de ces composés ont fait l'objet de recherches fondamentales et appliquées. On possède relativement peu d'information concernant l'influence de ces traitements sur l'insaponifiable de la matière grasse butyrique.

Lors d'une étude systématique des stérols, il a été démontré que les modifications sont plutôt d'ordre quantitatif et non qualitatif (Huyghebaert [1]). La distribution des stérols dans les diverses fractions, obtenues à partir de la matière grasse, est déterminée par leur localisation dans le lait.

Quelques aspects de l'influence des procédés de raffinage sur la composition des stérols de l'huile de beurre sont décrits dans la littérature. Guyot et Forceilles [2] ont discuté de l'incidence de l'hydrogénation sur les stérols, les alcools et les hydrocarbures. La formation d'esters de stérols lors de l'interestérification a été étudiée par Huyghebaert et Hendrickx [3]. Le 3,5-cholestadiène a été identifié par Homberg [4] dans les beurres décolorés. Ces résultats sont confirmés par Kuzdzal-Savoie et al. [5], qui proposent le 3,5-cholestadiène comme marqueur de raffinage des graisses animales.

Lors d'une étude concernant l'incidence des techniques fromagères sur la composition de l'insaponifiable, nous avons démontré la présence d'un stérol normalement absent dans les stérols du lait ; la présence de ce stérol a été observée dans les fromages à pâte molle moisie, affinés à l'aide de $P$. caseicolum. Son isolation et son identification comme de l'ergostérol font l'objet de cette publication.

\footnotetext{
* Laboratoire de Chimie Alimentaire. Faculté des Sciences Agronomiques. Université de Gand, Coupure 533, B-9000 Gand (Belgique).
} 


\section{METHODES}

Les fromages examinés sont des fromages d'origine belge et étrangère, achetés sur le marché local comme fromages prêts à la consommation.

Le fromage à pâte molle moisie, type Camembert, dans lequel nous avons étudié la biosynthèse de l'ergostérol et les phénomènes de maturation, est un produit courant d'une laiterie belge. Les souches de Penicillium caseicolum ont été isolées de plusieurs fromages; d'autres souches ont été reçues de firmes spécialisées.

La matière grasse est obtenue par extraction avec un mélange d'éther diéthylique et d'éther de pétrole après addition de $\mathrm{HCl}$ et chauffage en milieu acide.

Les stérols ont été isolés par précipitation avec la digitonine après saponification de la matière grasse butyrique. Les digitonides sont transformés en stérols libres ou en acétates. La teneur en stérols est calculée à partir de la teneur en digitonides et est exprimée sur la matière grasse.

Plusieurs méthodes ont été appliquées pour l'analyse des stérols. L'examen par chromatographie en phase gazeuse a été effectué sur trois colonnes de polarité différente : 3 p. 100 UCC-W98, 3 p. 100 OV17 et 3 p. 100 QFl. La technique de chromatographie sur couche mince a été appliquée pour la séparation des stérols sur gel de silice et sur gel de silice imprégné de nitrate d'argent.

La maturation a été suivie lors d'une fabrication industrielle par des déterminations du $\mathrm{pH}$ et de la matière sèche. L'intensité de la protéolyse a été déterminée par la mesure de teneur en substances azotées, précipitées par $\mathrm{KAl}\left(\mathrm{SO}_{4}\right)_{2}$, c'est-à-dire la teneur relative en caséine (Schulz [6]). La teneur en acides gras libres a été utilisée comme mesure de la lipolyse.

\section{PARTIE EXPERIMENTALE}

\section{III.1 La fraction stérolique des fromages}

L'examen des stérols, isolés de plusieurs fromages, a démontré que la composition correspond avec les compositions rencontrées pour la matière grasse butyrique (Huyghebaert [1], Guyot [7]). La teneur en stérols totaux ainsi que les teneurs en cholestérol et en stérols mineurs sont normales pour la matière grasse butyrique (tab. 1). Les résultats obtenus pour 200 échantillons de beurre sont donnés pour indiquer les variations normales. Le pic 1 est composé de 7-déhydrocholestérol et de latostérol; le pic 2 a été identifié 
TABLEAU 1

Teneur et composition des stérols des fromages (en p. 100 des stérols totaux)

\begin{tabular}{|c|c|c|c|c|c|}
\hline Type & Cholestérol & Pic 1 & Pic 2 & Pic 3 & Pic 1 a \\
\hline Pâte fraîche & 97,46 & 1,80 & 0,66 & 0,08 & \\
\hline Pâte fraîche, légèrement affinée & 97,55 & 1,47 & 0,91 & 0,07 & \\
\hline Pâte molle, type Camembert & 96,84 & 1,00 & 0,36 & 0,10 & 1,70 \\
\hline Pâte molle, type Herve & 98,73 & 0,81 & 0,37 & 0,09 & \\
\hline Pâte persillée, type Danablu & 99,02 & 0,59 & 0,34 & 0,05 & \\
\hline Pâte persillée, type Roquefort & 98,88 & 0,86 & 0,20 & 0,06 & \\
\hline Pâte pressée, type Gouda & 99,19 & 0,57 & 0,24 & $\operatorname{tr}$ & \\
\hline Pâte cuite, type Emmental & 98,77 & 0,70 & 0,50 & 0,03 & \\
\hline Pâte cuite, type Parmesan & 98,27 & 1,00 & 0,54 & 0,19 & \\
\hline Pâte molle, fromage de chèvre & 98,51 & 1,12 & 0,34 & 0,03 & \\
\hline Matière grasse butyrique & 97,95 & 1,66 & 0,32 & 0,07 & - \\
\hline 200 échantillons & $96,45-98,65$ & $0,87-2,10$ & $0,24-0,45$ & traces- 0,18 & \\
\hline
\end{tabular}


comme du 24-méthylènecholestérol, le pic 3 comme du 24-éthylidène cholestérol. Le pic du cholestérol comprend également des traces de 22-déhydrocholestérol et de 24-déhydrocholestérol (Huyghebaert [1]).

Ces valeurs démontrent que les modifications dans la fraction stérolique lors de la maturation des fromages sont très réduites pour des fromages affinés sous des conditions extrêmement variées.

Les fromages à pâte molle moisie type Camembert et similaire, forment une exception. Le pic 1 a, représentatif d'un composé typique, a été mis en évidence en quantités variables, dans tous les échantillons de fromages à pâte molle moisie affinés par $P$. caseicolum (fig. 1).
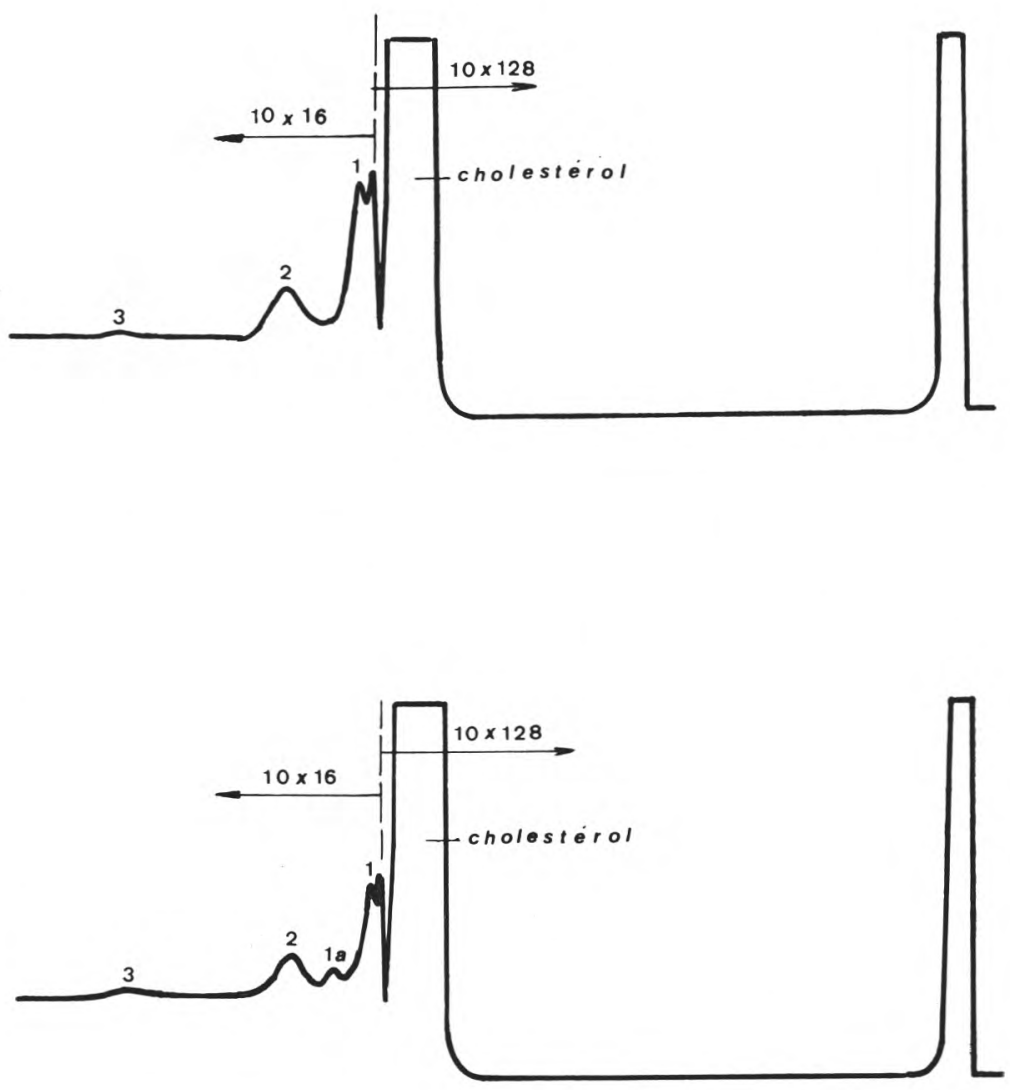

fig. 1

Chromatogrammes des stérols isolés de la matière grasse des beurres et des fromages. 


\section{III.2 Identification de l'ergostérol}

Le pic 1 a a été identifié comme de l'ergostérol; ce stérol n'a pas été décelé dans la matière grasse butyrique, même après enrichissement des stérols insaturés.

Les observations suivantes permettent de conclure que le composé est un stérol :

- la formation de digitonides, donc la précipitation par de la digitonine ;

- la libération des stérols libres à partir des digitonides par des solvants polaires;

- l'acétylation des digitonides et des stérols libres par l'anhydride acétique.

Les stérols, sous forme d'acétates, ont été analysés par chromatographie gazeuse sur trois phases liquides différentes. Le temps de rétention mesuré relativement par rapport à celui du cholestérol, était 1,19 sur les colonnes UCC-W98 et QF1 et 1,31 sur la colonne OV17. On obtient exactement les mêmes valeurs pour l'acétate d'ergostérol de référence.

Les stérols libres ont été séparés sur couche mince; on effectue une séparation des $\Delta 5$ et $\Delta 7$ stérols sur gel de silice; la structure $\Delta 5$, du pic 1 a a été confirmée.

L'insaturation du pic 1 a été démontrée par chromatographie sur colonne de gel de silice, imprégné de nitrate d'argent. Le pic 1 a et l'ergostérol sont retrouvés dans la fraction tri-insaturée.

Ces propriétés chimiques permettent d'identifier le pic 1 a comme de l'ergostérol.

\section{III.3. Présence et localisation de l'ergostérol dans les fromages à pâte molle moisie}

Le tableau 2 donne les résultats obtenus lors de l'analyse de plusieurs échantillons de fromages. Ces fromages d'origine belge, française et allemande étaient toujours affinés par $P$. caseicolum. On y trouve évidemment des teneurs variées en ergostérol. La présence d'ergostérol semble être liée à $P$. caseicolum; la teneur dépend de plusieurs facteurs entre autres le stade de maturation, le type de fromage, le développement des moisissures et les propriétés de la souche.

La distribution de l'ergostérol dans le fromage a été étudiée. Les différentes parties en renferment des quantités très variables. La partie centrale crémeuse, constituant le cœur du fromage contenait 0,29 p. 100 de stérols parmi les lipides totaux mais l'ergostérol n'était pas décelable. Par contre la croûte contenait 0,33 p. 100 de 


\section{$T A B L E A U$}

Teneur et composition des stérols, isolés de la matière grasse butyrique des fromages à pâte molle, affinés par $P$. caseicolum

\begin{tabular}{|c|c|c|c|c|c|c|}
\hline Echantillons & 1 & 2 & 3 & 4 & 5 & 6 \\
\hline $\begin{array}{l}\text { Teneur en stérols : } \\
\mathrm{g} / 100 \mathrm{~g} \text { lipides }\end{array}$ & 0,29 & 0,30 & 0,27 & 0,26 & 0,29 & 0,30 \\
\hline $\begin{array}{l}\text { Compositions des sté- } \\
\text { rols (p. 100) : }\end{array}$ & & & & & & \\
\hline Cholestérol & 97,19 & 87,82 & 92,18 & 96,04 & 93,77 & 90,96 \\
\hline 1 & 1,11 & 1,32 & 1,25 & 1,60 & 1,34 & 1,27 \\
\hline 2 & 0,60 & 0,39 & 0,47 & 0,46 & 0,51 & 0,38 \\
\hline 3 & 0,23 & 0,17 & 0,11 & 0,06 & 0,10 & 0,07 \\
\hline Ergostérol & 0,87 & 10,30 & 5,99 & 1,84 & 4,28 & 7,32 \\
\hline
\end{tabular}




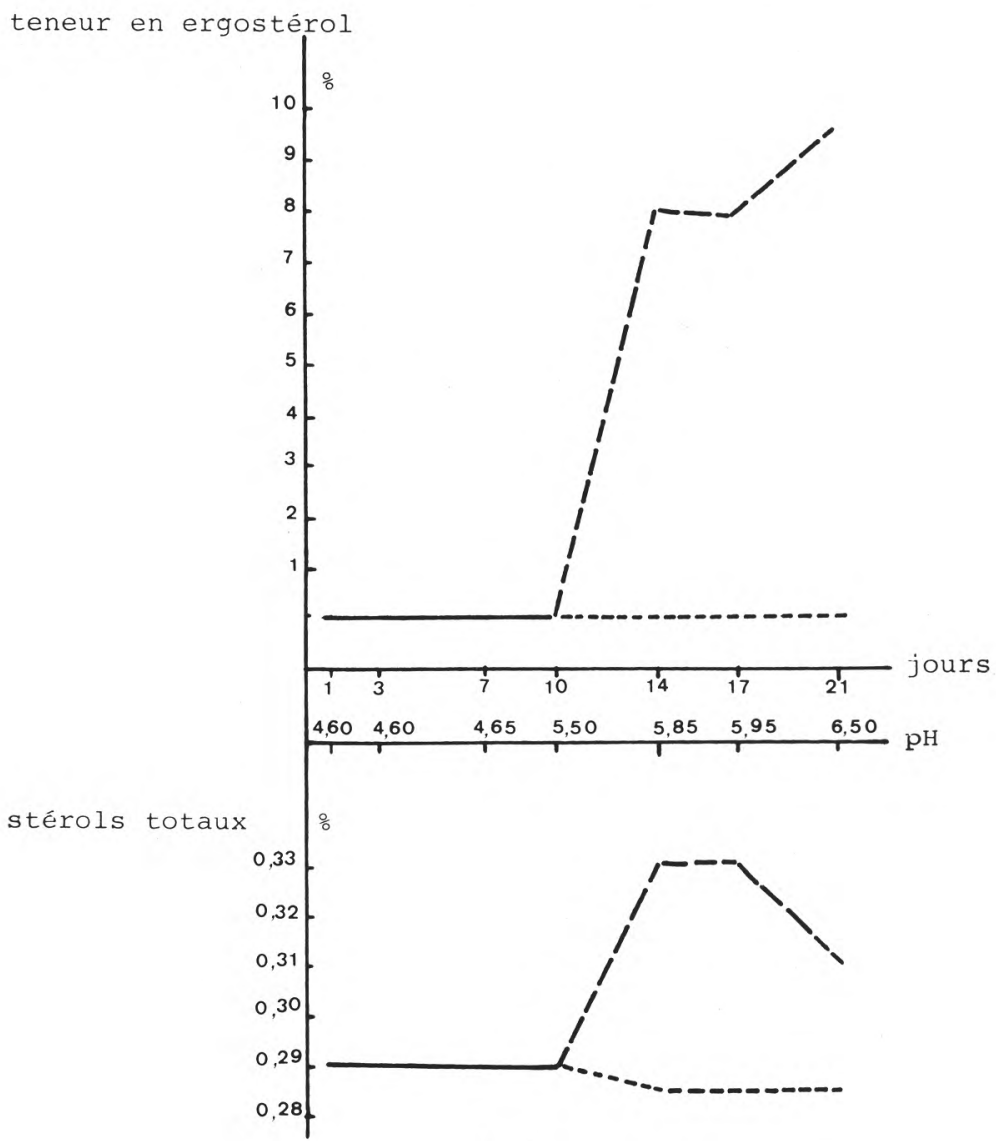

fig. 2

Teneur en stérol et en ergostérol lors de l'affinage

- fromage entier.

- - - partie extérieure.

-. . . - partie intérieure.

stérols dans la matière grasse, mais la teneur en ergostérol s'élevait à 8,5 p.100 des stérols totaux.

La présence d'ergostérol est liée au mycélium de $P$. caseicolum. Contrairement au phénomène observé pour les enzymes responsables de la maturation des fromages (Lenoir [8]) il n'y a pas de migration de l'ergostérol vers l'intérieur du fromage. 


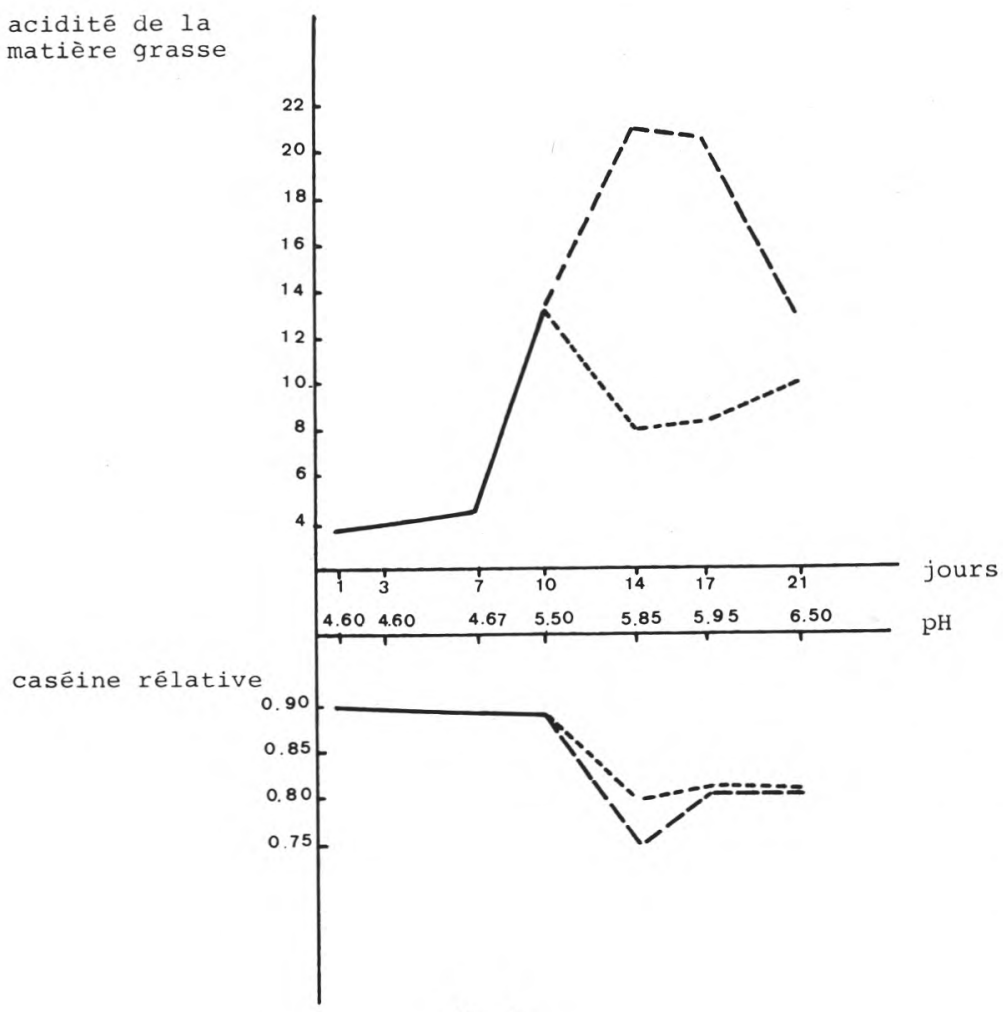

fig. 3

Protéolyse et lipolyse lors de l'affinage des fromages étudiés. fromage entier.

- - - partie extérieure.

-... - . partie intérieure.

\section{III.4. Biosynthèse de l'ergostérol lors de l'affinage du fromage}

La formation d'ergostérol en relation avec les phénomènes de maturation a été étudiée lors de la production de Camembert. Des échantillons ont été prélevés à plusieurs stades de l'affinage. Dès que possible le fromage fut divisé en deux parties : la partie intérieure ou le cœur et la partie extérieure ou la croûte.

La figure 3 donne l'évolution de la lipolyse et de la protéolyse lors de l'affinage. La lipolyse, exprimée en acidité, est appréciable après $10 \mathrm{j}$ tandis que la protéolyse, exprimée en caséine relative, 
devient importante après $14 \mathrm{j}$. Le début de la protéolyse se situe un peu plus tard que celui de la lipolyse. Il est évident que ces phénomènes sont plus prononcés dans la partie extérieure du fromage.

La teneur en stérols totaux dans la matière grasse ainsi que la teneur en ergostérol dans les stérols sont données dans la figure 2. Le début de la formation d'ergostérol se situe entre 10 et $14 \mathrm{j}$ d'affinage. L'ergostérol est retrouvé uniquement dans la couche extérieure. Après être passé par un maximum le taux d'ergostérol dans les stérols totaux a tendance à diminuer, étant donné que la couche crémeuse devient plus épaisse. Il existe un parallélisme entre la biosynthèse de l'ergostérol et les phénomènes de maturation.

La relation entre les augmentations des teneurs en stérols et en ergostérol permet de supposer que l'augmentation de la teneur en stérols est exclusivement due à la biosynthèse d'ergostérol. On peut pertinemment conclure que l'ergostérol est formé de novo et non pas à partir de cholestérol. Cette conclusion est confirmée par les essais suivants.

\section{III.5. Biosynthèse de l'ergostérol dans des systèmes modèles}

L'hypothèse que l'ergostérol est synthétisé par $P$. caseicolum et n'est pas un produit de transformation du cholestérol est basée sur :

- la localisation de l'ergostérol dans la couche extérieure du fromage ;

- le fait que l'augmentation du taux de stérols totaux est due à la biosynthèse de l'ergostérol.

Néanmoins le cholestérol serait toujours un produit intermédiaire dans le cycle de la biosynthèse de l'ergostérol (Templeton, 9).

Dans le but de confirmer cette hypothèse, des milieux exempts de cholestérol ont été utilisés comme substrats et on a fait varier la composition de ceux-ci. Les taux d'ergostérol les plus élevés ont été obtenus sur un milieu qui s'approche de la composition d'un caillé salé exempt de matière grasse. Ce milieu était composé de :

- 20 p. 100 de caséinate de sodium;

- 3 p. 100 de $\mathrm{NaCl}$;

- d'acide lactique jusqu'à obtention d'un $\mathrm{pH}$ de 5,5.

L'absence de cholestérol dans le milieu a été vérifiée par chromatographie gazeuse. Les quantités de stérols obtenues avec deux souches sont données dans le tableau 3 . La souche 1 est une souche couramment utilisée en fromagerie. La souche 2 a été isolée d'un fromage de commerce. De ce tableau, il résulte d'une part, que les deux souches sont productrices d'ergostérol et de cholestérol, d'autre part, que le cholestérol de la matière grasse butyrique n'est pas un élément indispensable pour la biosynthèse de l'ergostérol. 


\section{TABLEAU 3}

Biosynthèse du cholestérol et de l'ergostérol sur un milieu exempt de matière grasse butyrique

\begin{tabular}{|c|c|c|c|}
\hline & $\begin{array}{l}\text { Incubation } \\
\text { (j) }\end{array}$ & $\begin{array}{l}\text { Mg d'ergostérol } \\
\text { dans } 100 \mathrm{~g}\end{array}$ & $\begin{array}{l}\mathrm{Mg} \text { de cholestérol } \\
\text { dans } 100 \mathrm{~g}\end{array}$ \\
\hline Souche 1 & $\begin{array}{r}0 \\
7 \\
14 \\
21\end{array}$ & $\begin{array}{l}\overline{0,11} \\
0,20 \\
0,26\end{array}$ & $\begin{array}{l}\overline{1,32} \\
4,62 \\
6,59\end{array}$ \\
\hline Souche 2 & $\begin{array}{r}0 \\
7 \\
14 \\
21\end{array}$ & $\begin{array}{l}\overline{-} \\
0,09 \\
0,34 \\
0,90\end{array}$ & $\begin{array}{r}-\overline{0,83} \\
6,57 \\
21,25\end{array}$ \\
\hline
\end{tabular}

\section{CONCLUSION}

L'ergostérol ou provitamine D (ergosta-5, 7, 22-triène-3 $\beta$-ol) a été mis en évidence dans tous les fromages examinés, affinés à l'aide de moisissures superficielles du type $P$. caseicolum. La présence de ce composé non encore décelé dans une matière grasse butyrique ordinaire, est liée au mycélium de cette moisissure. Contrairement aux enzymes, une migration de l'ergostérol vers l'intérieur du fromage n'a pas été observée; il s'agit donc d'un composé intracellulaire.

L'étude de la biosynthèse de l'ergostérol lors de l'affinage a permis de démontrer que le début de sa formation se situe au moment où l'activité lipolytique et protéolytique deviennent appréciables.

Au cours d'essais effectués sur des milieux exempts de cholestérol, il a été démontré que le cholestérol de la matière grasse butyrique n'est pas un substrat indispensable pour la biosynthèse. Les souches de $P$. caseicolum sont productrices d'ergostérol et de cholestérol, le cholestérol étant un produit intermédiaire de la biosynthèse de l'ergostérol.

\section{Remerciements}

Nous remercions vivement MM. J. Fovel et L. Desmijtere de leur précieuse collaboration. 


\section{R és u mé}

L'ergostérol, un précurseur de la vitamine $\mathrm{D}_{2}$, normalement absent dans les stérols de la graisse butyrique, a été démontré dans des fromages à pâte molle moisie (Camembert et autres). La présence a été confirmée par examen des subtances de référence, par chromatographie gazeuse et sur couche mince.

L'ergostérol a été retrouvé dans la partie extérieure du fromage. La teneur augmente parallèlement avec la protéolyse et la lipolyse. Le processus de la maturation dans une production industrielle et l'étude des systèmes modèles a démontré que l'ergostérol est synthétisé par $P$. caseicolum pendant la maturation et que le cholestérol n'est pas un substrat essentiel pour la synthèse d'ergostérol.

\section{S u $\mathrm{m}$ m a r y}

Ergosterol, a precursor of vitamine $\mathrm{D}_{2}$, normally absent in the sterols of milkfat, has been demonstrated in white surface mold cheese (Camembert and others). The presence has been confirmed by comparison of reference substances, by gaschromatography and by thin layer chromatography. Ergosterol has been found in the outer layer of the cheese. There is a good agreement between the ergosterol content and the degree of proteolysis and lipolysis. The formation during ripening in an industrial production and the study in model systems has demonstrated that ergosterol is formed by $P$. caseicolum during ripening and that cholesterol is not an essential substrate in the ergosterol synthesis.

Reçu pour publication en juin 1979.

\section{Bibliographie}

[1] Huyghebaert (A.) (1976). - Thèse d'agrégation, Université de Gand.

[2] Guyot (A. L.) et Forcerlle (M. J.) (1976). - Détermination de l'hydrogénation de la matière grasse butyrique par l'analyse de l'insaponifiable. Actes du $13^{e}$ Congrès Mondial de I.S.F., Marseille, B-61.

[3] Huyghebaert (A.) et Hendrickx (H.) (1976). - Esters de stérols dans les corps

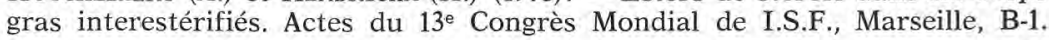

[4] Homberg (E.) (1975). - Veränderung der Sterine durch industrielle Verarbeitungsprozesse von Fetten und Oelen. Fette Seifen Anstrichmittel, 77, 8.

[5] Kuzdzal-Savoie (S.), Langlois (D.) et Krobicka (A.) (1978). - Le cholestadiène3,5 marqueur de raffinage des graisses animales. Communication, $20^{\circ}$ Congrès International de Laiterie, Paris, 373. 
[6] Schulz (M. E.) (1953). - Der relative Kaseingehalt von Käse. 13th International Dairy Congress, 's Gravenhage, 2, 576.

[7] Guyot (A. L.) (1968). - Détermination des falsifications du beurre par certaines graisses transestérifiées. Bull. Rech. Agron. Gembloux, 3, 128.

[8] Lenoir (J.), Glenza (A.), Bergère (J. L.), Cerf (O.), Choisy (C.), Desmazeaud (D.) et Hermier (J.) (1973). - Les facteurs de production du système protéolytique de Penicillium caseicolum. Le Lait, 53, 246.

[9] Templeton (W.) (1969). - An introduction to the chemistry of terpenoids and steroids. Butterworth London. 